\title{
Responding to COVID - 19: Insight Into Capability Re-Configuration of Healthcare Service Ecosystems? The Use Case of Hospitalization at Home
}

\author{
Nabil Georges Badr \\ Saint Joseph University \\ Luca Carrubbo \\ Salerno University \\ Margherita Ruberto \\ Salerno University
}

\begin{abstract}
An effective Healthcare Service Ecosystem must emphasize the notion of well-being co-creation which entails a dynamic interplay of actors, in face of the challenges, with their ability to use the available resource pools, at the different system levels. An appropriate response, largely avoiding any crisis, depends on a society's resilience and the related response of actors in the reconfiguration of resources. Originally considered luxury and for the fortunate few who could afford the learning curve, Hospitalization-at-Home (HaH) recently approached a new normal with a positive impact to health outcomes. Nowadays, hospitals have had to reconfigure their health services to reduce the workload of caregivers during the COVID-19 outbreak. We show an example of how HaH can be a viable re-configuration of Healthcare Service Ecosystems and a use case for capability reconfiguration. Our use case can be a lesson for the adaptation of technology for patient empowerment allowing patients to interact with their care ecosystem while at their home.
\end{abstract}

Keywords: hospitalization at home, systems thinking, viable system, complex ecosystems, healthcare service ecosystems

\section{INTRODUCTION}

An effective Healthcare Service Ecosystem (H-SES) (Frow et al, 2014) must emphasize the notion of well-being co-creation which entails a dynamic interplay of actors, in face of the challenges, with their ability to use the available resource pools, at the different system levels (Häring et al, 2017).

In pandemics, an essential healthcare disaster per sort, the social as well as the service-related fabric of society, supply chains (Bonadio et al, 2020) and even the complete industry are changed. An appropriate response, largely avoiding any crisis, depends on a society's resilience and the related response of actors in the reconfiguration of resources (Finsterwalder \& Kuppelwieser, 2020). Healthcare systems are no exception (P2PH, IHI.ORG). "The COVID-19 era is bringing new attention and urgency to people's social 
needs, the impact of unmet needs on health, and the importance of partnership among health systems and community organizations" (P2PH, IHI.ORG).

New behaviours have to be learned for society to maintain the well-being of its constituents and new processes put in place and for the survival of the multiple, and the sustainability of related ecosystems in support of society. Healthcare Service Ecosystems have to support new concepts and services; technology deployment that facilitates telemedicine, care at home and consultation at a distance must be accelerated to expand the public's access to essential health services during the COVID-19 Pandemic (CDC.ORG).

\section{Motivation}

Healthcare systems have buckled under the health emergency in the pandemic, due to insufficient hospital availability of beds, long waiting times, lack of adoption of intervention plans for emergencies, lack of medical and health personnel, of the total absence of coordination between the different ones' actors and, above all, the lack of an adequate assistance territorial network (Grimm, 2020). Furthermore, the delay in the digitization process and the presence of IT systems fragmented made it difficult to exchange information with serious repercussions on timeliness of the implementation of all control measures.

For practitioners, the sudden unbalance between health care needs and available resources has confronted health professionals' ethical choices with the need to make decisions in time very short, paying a high toll also in terms of human lives (Baily et al, 2007).

For patients, the pandemic has confined the population to their homes. Those ridden with chronic illnesses must have access to supervised and continued care. In all, medical healthcare providers are under an enormous amount of workload pressure, faced with high risks and shortages of available services, along with increased total health expenditure (Moazzami et al, 2020).

This situation underscores the importance of a reorganization of health services and can be seen as the perfect storm, thus motivating healthcare ecosystems to include care at home technologies in their mainstream. Aside from building responsive information systems for a timely collection of information for timely and relevant decision-making, a paradigm shift in care models must rely on the empowerment of territorial health care aimed at an effective taking charge of patients both in terms of appropriateness of care and clinical governance (Breslow et al, 1992).

Originally considered luxury and for the fortunate few who could afford the associated learning curve, Hospitalization-at-Home (HaH) (Leff, 2001) approached a new normal with a positive impact to health outcomes. A recent study found that substitutive home hospitalization not only reduce cost by $38 \%$, by improved patient experience. At the comfort of their home, patients spent a smaller proportion of the day sedentary and were readmitted less frequently within 30 days ( $7 \%$ vs. $23 \%)$, mainly due to the lack of potential infections risks, otherwise extant in traditional hospital settings (Levine et al, 2020). Clinicians are leading service reconfiguration to cope with COVID-19. They are learning new skills, adapting and exploiting new means of consultations, like the use of video clinics (Thornton, 2020) for example. In other cases, hospitals have had to reconfigure their health services to reduce the workload of caregivers during the COVID-19 outbreak, such in the case of the deployment of easy to use software / devices that allow patients to interact with their care ecosystem while at their home. In general, direct-to-consumer telemedicine products, for instance, can enable patients to connect with their healthcare provider at a distance. This indicates that Healthcare ecosystems have had to learn to reconfigure their resources.

Therefore, "HaH can be viewed as a practical expression of H-SES adaptive features, re-configuration ability and modular design on the grounds of the System Thinking perspective". The adaptation of processes and technology can empower patients to interact with their care ecosystem while at their home.

\section{What Can We Learn From a Hospitalization at Home Use Case Regarding the Capability Re-Configuration of Healthcare Service Ecosystems?}

To illustrate our thinking, we formed this manuscript under the lens of systems thinking applied to Service Ecosystems (Section 2.1) of healthcare with the example of $\mathrm{HaH}$ as evidence (Section 2.2) and posturing the value of technology as the central component (Section 2.3) with a use case that treats the topic (Section 3) and draws some challenging issues (Section 4). 


\section{BACKGROUND}

\section{Healthcare Service Ecosystems}

Starting from the recent advance in service research on H-SES, a number of reflections have been synthetized in terms of the actors' engagement and participation (Frow et al, 2019), including formal and informal caregiver interactions (Badr et al, 2018). Special attention has been given to the design and reconfiguration, adaptive processes, and the ability to face the emergence in the systems (Capunzo et al, 2013; Carrubbo et al, 2013; 2016; Ciasullo et al, 2017).

Essentially, Healthcare Service Ecosystems can be viewed as complex service ecosystems (Ciasullo et al, 2017), due to the distinctive features and the ability the re-configure fundamental resources in reaction or anticipation of external events, where decision-makers have to manage complex interactions between several different actors or entities (e.g., patients, health providers and suppliers, etc.). Through the lens of system thinking, the capability of reconfiguration of multiple resources to deliver value in a different modality requires an adaptive, cognitive alignment for maintaining the system's viability; i.e. the ability of Actors in the system to 'continue' their actions and survive the impending events. systems that aim to survive in their living context by establishing harmonic relationships with other entities that own the resources necessary for their functioning and survival (Barile et al, 2012a; 2012b; 2016). All parts of the system are interconnected and interact with each other, providing continuous feedback that serves as a learning cycle for capability reconfiguration in response to a certain event. Inherent in these dynamic capabilities of reconfiguration is a level of complexity (variety, variability, indeterminacy). For example, community first responder programs can improve the speed of access to resources in the time of need, with faster interventions that could improve care outcome by expediting patient care. Additionally, including informal care givers in the system of care helps leverage off elastic levels of resources that would relieve the demand off the system, improve community sustainability and co-create value (Sorrentino et al, 2017). Point of care coordination programs use platforms and advanced analytics applications to improve accuracy and timeliness of data for informing decision making and monitoring the performance of the complex heatlh service ecosystem. As part of this ecosystem, just-in-time adaptive interventions (JITAI) for example provide the right type/amount of support, at the right time, through the availability of increasingly powerful mobile and sensing technologies (Nahum-Shani et al, 2018). The pervasiveness of the adaptability paradigm has pressed legal and non profit organizations to advocate the need for certain changes to laws and regulations that indirectly influence adjustments to the system of care for a continuously adaptable ecosystem.

\section{Hospitalization-at-Home}

$\mathrm{HaH}$ is one approach for a H-SES to solve problems and adapt to evolving contingencies, transforming the model of care. HaH is based on the implementation of alternative forms for health assistance such as healthcare residences, home care, intermediate care, community mail, and weekend shipments (Caputo, 2018). From a more general perspective, the main value proposition of $\mathrm{HaH}$ is the reduction in the number of hospitalizations and the related reduction of hospital care costs and clinical risks (Hwang et al, 2008). The changes in care settings (Al-Balushi et al, 2014), and on the cost reduction for managing hospital health processes (Bodenheimer et al, 2020), are also indicative of this transformation.

To achieve this aim, HaH proposes a redefinition (re-configuration) of the hospital as an advanced place of care, and it underlines the need for specific organizational paths, directly, so as to identify the procedures for collecting timely and up-to-date data about health services demand and the resources used during the health processes. This implies new modes to intend value, influenced by contextualization (value-incontext), personal patient's use (value-in-use) and their own direct experiences (value-in-experience) (Polese et al, 2018) as they have been exploited by service scholars worldwide in last dacades. The three pillars of economy, society, and environment that define of sustainability science, have inspired principles of efficiency of healthcare service, effectiveness in resource usage, and patients' satisfaction. These principles can be levers for promoting reconfigurable healthcare processes (ibidem). 
Firstly, HaH can be considered as an alternative approach to consolidated health treatments and it can increase efficiency in the use of the available resource. Expanding the value to different contexts of the ecosystem, $\mathrm{HaH}$ clinical activities are managed both at a local (hospitals and districts) and regional level; the activities of diagnosis, treatment, monitoring, and rehabilitation are provided within several constraints in care quality (i.e., waiting time), efficiency (i.e., resource utilization), and costs (i.e., fixed annual savings or budget reduction) (Ignone et al, 2013). Difficulties in obtaining comprehensive assessments have spawned the innovation of self-contained $\mathrm{HaH}$ service packages, to monitor changes in health status, while working within existing funding constraints.

Secondly, thanks to this alternative approach to healthcare processes, $\mathrm{HaH}$ may be credited for satisfying the collective need for health for reducing the health processes costs. HaH expands care processes to the setting of the patient and their informal caregivers. It is not a trivial initiative as it stretches the boundaries of the classical care and the workflows and processing into an integrative formula that extends beyond the classical dyadic relationships between provider and patient. The HaH system needed to adapt the processes, actions and interactions over a larger sphere that what would be in a traditional hospital setting. These processes need to be design to help overcome hurdles of arranging care, following treatment regimens and controlling symptoms. Away from the traditionally controlled flow mechanisms (Badr et al, 2019). The demand to coordinate care from a mix of providers puts a strain on a system that must reconfigure itself for the variance in constraints and variety of conditions. For instance, essential actors in the transformation of care in this complex ecosystem, informal caregivers have become the cornerstone of value exchange in the health ecosystem their contribution extend the platform of care and service to the patient's setting (Sorrentino et al, 2016).

Thirdly, HaH can positively impact on patients' quality of life. HaH introduces new forms of responsibility and engagement in the health domain, offering to patients and their families the opportunity to acquire the knowledge and competencies useful to proactively collaborate with health professionals (Rodríguez et al, 2013). Because it aims at organizing in the patient's home a "care setting" equivalent to the hospital one, helpful for chronic illnesses, $\mathrm{HaH}$ has the potential to increase patient and caregiver satisfaction so as to improve patients' quality of life. In the HaH setting, the patient is generally in a known comfortable setting. The experience is then borne out of interactions, communications and transactions, controlled by the patient, in the majority (Badr et al, 2019). That said, the gain in value of the patient's experience an also bear a new set of challenges that must be overcome, in the form of interaction with informal caregivers at home, through the use of domotic devices or other issues of technology literacy addressed in the next section.

In this context, the COVID Pandemic $\mathrm{HaH}$ can be the quality of life alternative for chronic patients who may need frequent treatment and find themselves otherwise subject to exposure without the HaH facilities and services. Our case study provides a testimonial to such enhanced experience.

Therefore, considering the multiple potential contributions provided by $\mathrm{HaH}$ for increasing the efficiency and sustainability of H-SES, several approaches have been proposed for evaluating its dynamics, focusing attention on the decrease in hospitalizations (Cohen et al, 1996), hence, bolstering the overall value-in-experience of this extended form of care. Through hospitalization at home, H-SES can reconfigure itself and re-organize itself for increasing the capability of the multiple needs of patients (Polese, Carrubbo, 2016). In a nutshell, according to Wilson (2018), the most relevant advantages provided by HaH practice can be summarized by provisioning components of interdisciplinary team-based community care as part of integrated care with other sectors, bridging restorative approaches to care with the support for caregivers as part of home care (Polese et al, 2018).

\section{The Emergence of Telemedicine and Assistive Devices}

At the centre of the reconfiguration capability of the H-SES, health technology should be patient centric, and focus on the interaction between the patient and the multiple actors and services in the healthcare ecosystem. Inevitably, Internet of Medical Things (IoMT) devices are being exploited for accurately and readily measure different aspect of human life with the intent to learn and improve on its outcome. Internet of Medical Things (IoMT) refer to the collection of medical devices and applications that 
connect to healthcare IT systems through online computer networks, equipped with Wi-Fi that allow the machine-to-machine communication (Al-Turjman et al, 2020).

Effective solutions have been developed to manage the interaction among the care team (Badr et al, 2018), provide assistive functions (assistive technologies) and improve the patient's quality of life (Sofaer \& Firminger, 2005; Moliner, 2009; Sweeney et al, 2015). Through the deployment of point of use systems and software based on principles of communication, data management, patient engagement has become key to the expansion of the H-SES (Britt et al, 2005; Gruman et al, 2010; Polese \& Carrubbo, 2016). For instance, telehealth adoption is expanding the accessibility to healthcare service beyond the traditional setting, with services such as virtual consultation, allowing access to cost-effective care. Soon, telepresence physicians will use robots to help them examine and treat patients in rural or remote locations (ASME.ORG).

The increase of consumerism in remote healthcare devices is democratizing current healthcare systems. Consumer-driven care delivery models such as telehealth, e-pharmacy, retail care, price transparency, push care closer to the point of the person, among others. Examples are wearable devices such as heart monitors that can detect atrial fibrillation, blood pressure monitors, self-adhesive biosensor patches that track your temperature, heart rate, will help consumers proactively get health support. Drug delivery devices such as insulin pens, biologic auto injectors, inhalers, and smart packaging for pills will be commonplace to enhance both clinical and business operations in healthcare (https://flex.com/industries/healthcare)

Technology platforms will soon become permanent gears within the patient homes, which will reduce costs associated with hospital stays, increased ease, and comfort for patients, and minimizing exposure of clinical staff to infected patients (himss.org). However, such levels of exploitation of technology necessitates another vein of adaptation for the system to sustain itself in the HaH context. For example, the use of home-based assistive devices that would replace the hospital setting, would require a significantly flexible feature to support the wider range of patient potential disabilities affecting their ability to use domotic technology as opposed to the highly standardized traditional point of care hospital equipment. In order to maintain a positive patient experience, such systems must take into account the prior experience in using assistive technology and the patient's strengths and weaknesses in areas such as reading, writing, math, spelling, listening, memory, and organization. This may necessitate adequate training and encouragement to learn the required skills - or the provisioning of a technology support system, added on the health ecosystem (Badr et al, 2019).

Certainly, as an emerging technology, it is unrealistic to expect that solutions as such to be based on standardizations, given the high degree of heterogeneity of integrated care practices in place, and the impossibility of forecasting future demands for care. However, it is an indication that the H-SES is attempting to reconfigure itself through the use technology among others.

\section{Technology Applications for Integrated Care}

Central to the sustainability construct in a complex heath ecosystem is the notion of building and fostering partnerships and integration of care providers (Plochg et al, 2006). Such partnerships must leverage processes and technology available to support the continuity of the services through integration. The model of integrated care delivery becomes an essential component in the success of the HaH service.

We have seen the development of technology applications with features that enhance the value production of integrated care through functions that improve dialog, communication and coordination capabilities (Restifo et al, 2015). These applications aggregate data collected from different sources in the care ecosystem, and enable its analysis and translation into essential feedback/treatment information, and be accessible and easy to use for all healthcare ecosystem users. The potential of bridging the $\mathrm{HaH}$ ecosystem with its traditional one building-centric care system hence relies on the integration of all these components at the service of the human actors in the system, in order to complete the value chain and deliver home based care. 


\section{HAH EXPERIMENTAL USE CASE}

Here, we introduce an interesting use case exemplifying reconfigurable Healthcare Ecosystems. The setting of our example is in South of Italy, in Salerno City (Campania Region).

\section{The 'ADD-Protection' Co-Financed R\&D Project}

The Project was named 'ADD-Protection' to mean the increase of care provision to defend the health of the community, additional to the traditional protocol already existing. This attempt was before COVID19 situation, but still represent a best practice to efficiently respond to uncertain and unpredictable conditions that can occur over time. Results explain how the innovative solution proposed can effectively support a new organization and design of healthcare service (seen as a whole) when needed, and today give us (scholars, practitioners, medical employees, managers and politicians) a very relevant suggestion to perform a continuative care in the unusual and we are living now.

The Project was about 1 year long and gave lots of insightful information about the problems and the opportunities to improve the performance level and quality by offering modular technology solutions with a high potential to enable evolutions in the global Healthcare Service Ecosystem as a whole.

The experiments involve 50 persons ( $40-85$ years old) affected by 3 special pathologies: diabetes in adults, heart difficulties, breath chronical problems.

\section{The 'ADD-Protection' Research Activities}

Prior to the COVID-19 pandemic, The Hospital of Salerno, named San Giovanni di Dio Ruggi d'Aragona in San Leonard - launched an experiment in collaboration with SIMAS Intedept., a research Centre of Salerno University and a local firm Magaldi Life Ltd. The attempt was to develop a new protocol to evaluate specific cases of chronic disease of long-term patient, in the aim to provide care to their homes.

The project included multiple milestones (Figure 1) incorporating the expansion of the definition of a multimode medical service protocol and communication plan, the development and implementation of an information system for remote medical examination and the supporting infrastructure. The technology implemented allowed for the monitoring of vital signs and the detection of early warning. The project also accounted for cost reduction measures, changes in the related processes, the diffusion of training to the actors in the service, including informal caregivers.

The following project plan was laid out to manage and monitor the activities in the following milestones:

- MIL_1 - Development of an election procedure for the definition of the perimeter of "appropriateness of protected discharge" in a logic that may include, alongside the dual option: HOSPITAL - HOME; a multipolar option: HOSPITAL - HOME and / or local RSA and / or HOSPICE.

- MIL_2 - Development of a systemic and multichannel communication plan through which the hospital structure informs its context around the value of the Protected Discharge model. The aim was to calibrate the awareness to improve the positive reception of the service and also removing all the elements of disinformation that it could reduce the effectiveness of the project to the extreme of its failure.

- MIL_3 - Development of a REMOTE MEDICAL EXAMINATION system aimed at activating a direct communication channel between patients and operators - in particular hospital doctors - which allows a level of continuity of care that is truly accessible by the patient, especially assessment of clinical conditions, functional and cognitive status.

- MIL_4 - Development of a dedicated IT platform for sharing information (knowledge management) between the parties involved in the service, with particular reference to: progress of the treatment plan, list of open problems, status of achievement of objectives, and improve level of satisfaction of the patient and family (process management information). 


\section{FIGURE 1 \\ MILESTONE PROJECT AREAS}

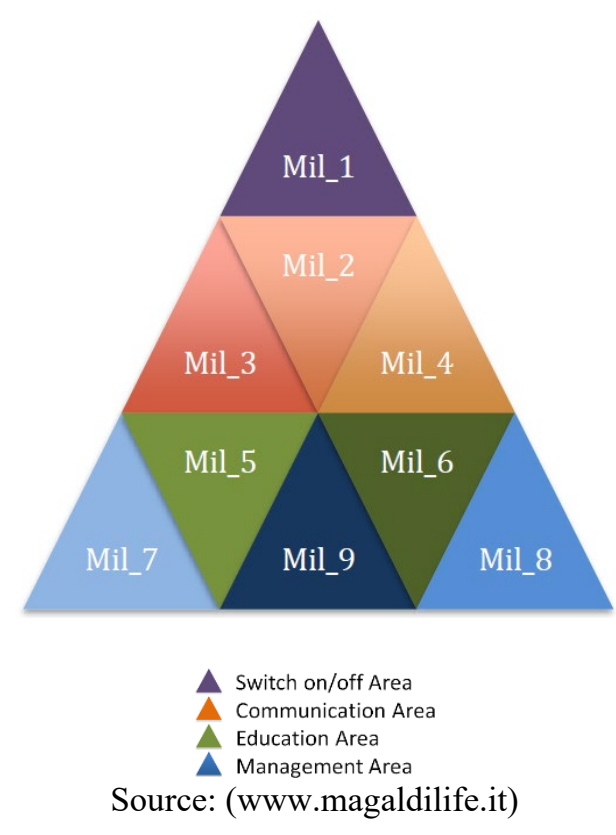

- MIL_5 - Development of new staff training (doctors and nurses) and any other actor involved in the service.

- MIL_6 - Development of a new approach to communication, involvement and active participation of discharged subjects and their families (informal care giver).

- MIL_7 - Development of an alert procedure that allows you to collect weak signals and weigh the risk factors that can lead to an early return to hospital, not limited to health factors related to the patient but also to those of family sustainability.

- MIL_8 - Development of a cost logic that goes towards the concept of "care budget", for the elaboration of an individual Care Plan ad hoc for the de-hospitalized patient.

- MIL_9 - Development of a renewed coordination of activities in accordance with the principles of Project Management, by virtue of the lower level of standardization of each intervention.

\section{The Technologies Used}

This $\mathrm{HaH}$ project was made possible by technologies such as electronic medical records, real-time diagnosis with go-pro cam, Big Data Analytics special tool, including the provisioning of infrastructures services required for the collection and treatment of data generated by the experiment.

Smart home and assistive devices integrated with a software interface. The interface at the patient's was modular, timely, easy-to-use, and compatible with all main existing information systems in Healthcare.

The supporting infrastructure relied on a cost effective cloud architecture, with a multi-channel for access (web, tablet, mobile) by all project stakeholders (OSS team, case manager, caregivers, project manager, etc.) to structured data and unstructured data captured on the platform. Interconnections with third parties (ERP HOSPITAL) and FSE (Electronic Health Record) provided the integration necessary for the information flow and coordination that allowed the possibility, in addition to reporting, to develop business intelligence algorithms, to obtain data and knowledge on a single practice and expand it to the exploit of the ecosystem.

For further reference, we have included, in the Appendix, Figure 2 depicting the overall set of components of the technology system used. 
FIGURE 2

THE NEW 'ADD' FLOW

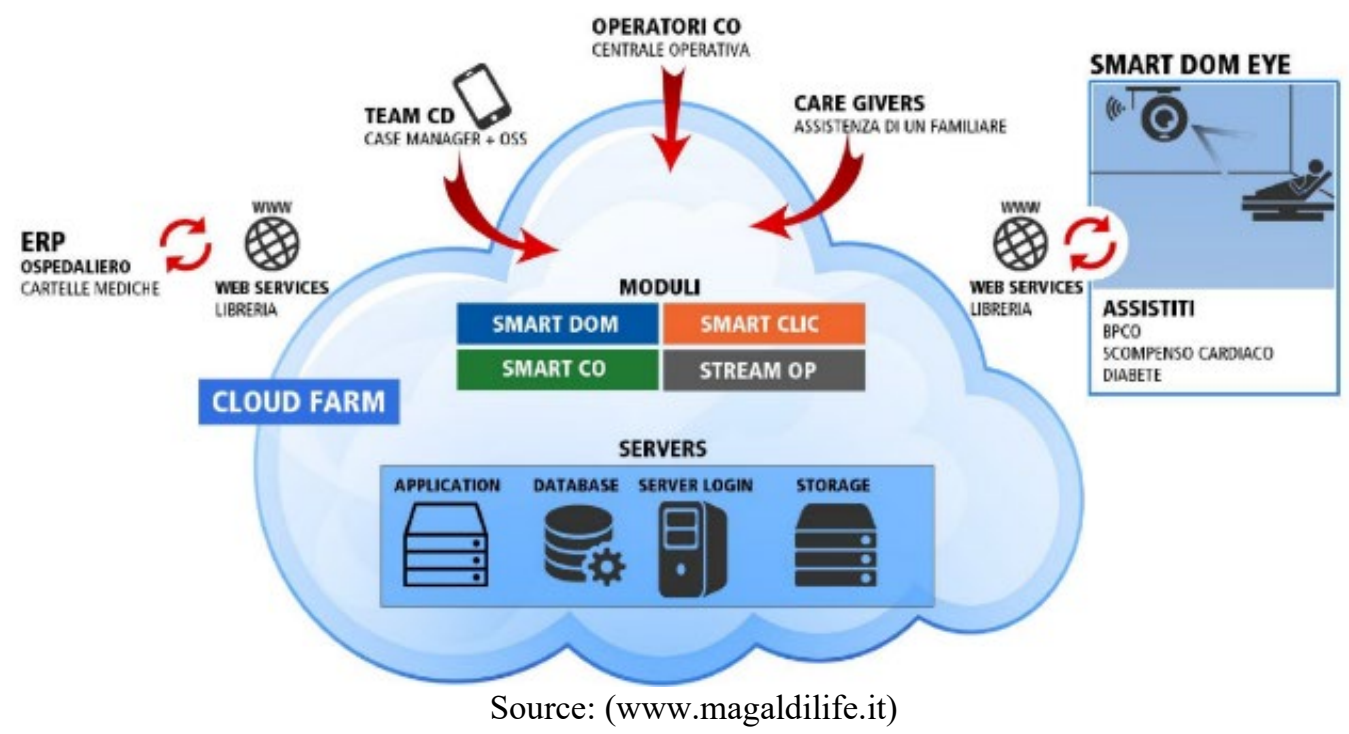

\section{OUTCOME AND REFLECTIONS}

\section{HaH Is a Viable Adaptation of a Healthcare Service Ecosystems}

The project was a success as it has bolstered the value of $\mathrm{HaH}$ as a viable model of care. As a result, the ADD project was sanctioned and the local level, into a set of HaH services.

Though initially apprehensive about the use of sophisticated technologies for care at home, the participants in the project viewed technology integration as an unalloyed benefit, as they cherish opportunities to be with loved ones at home rather than in a hospital, with the ability to quickly resume a normal life. Patients can progress their treatment plans outside the hospices of a hospital, reducing the care burden on the hospital staff, lowering the risk on the patient's health and significantly curbing the cost of care.

Effectively, the implementation of $\mathrm{HaH}$ services reduced the demand on the resources of the hospitals providing the same treatment to $\mathrm{HaH}$ patients at a lower cost, availing the resources of the hospital for inpatient care. Cost savings reported were to the scale of $870 €$ per bed per day (for 1 year).

Additionally, patients who participated in the ADD Protocol have reported better care and better experience (lessened need to visit the hospital) and practitioners have expressed better satisfaction due principally to reduction of their workload. Patients were empowered to address their issues and in the comfort of their environment; during the 1-year timeframe, $89 \%$ have reported better access to expert advice and punctuality in receiving care assistance and $95 \%$ had better hygiene. On the other hand, $93 \%$ of patients reported courteous and pleasant interaction with their care provider Patients reported with a higher quality experience overall.

Therefore, our use case has shown that $\mathrm{HaH}$, when done right, can,

(1) Improve effectiveness of care (e.g., lower readmission rates for heart failure patients);

(2) Reduce the threat of complications, for improved care outcome;

(3) Provide timely care - improving patient access to care;

(4) Increase the satisfaction of the variety of actors in the system (Patients, providers, etc.); and

(5) Reduce burden and cost on the Hospitals 


\section{HaH Is a Use Case for Capability Re-Configuration}

From the perspective of adaptation, we make evident how healthcare entities need to change and update protocols, procedures, operations, by following local needs, and adapting to physical, technological and infrastructural constraints. In our case, the use of technology underscored the advantage of integrating clinical actions with existing technical trends and tools. Lessons can be drawn for the implementation of a wider component of this ecosystem that solves for the crowding hospitals, in the seasonal peak of particular diseases, outside the structural limits of such a traditional hospital department, in the absence of doctors, medical teams or other personnel for any reasons.

Our case provides a perspective on how a healthcare ecosystem was reconfigured to deal with the variety, variability and uncertainty of the conditions in settings outside the perimeter of the traditional hospital. This capability reconfiguration was possible through reinforcing the sustainability of service provision, the lean management of resources (including people efforts), and the self-learning actions to implement the specialized know-how and skills/competences in care giving.

\section{Ethical Considerations to Ponder}

Such implementations bring forth a set of adoption and ethical issues to deal with. Historically, HaH has had faced a number of ethical, legal and clinical practice issues, at the levels of data-protection, patients' privacy, training of family caregiver, discharge planning, etc. (Arras \& ubler, 1994; Budd et al, 2020). The complexity of the phenomenon of high-tech home care has exposed patient data to be available to the occasional user of the devices at a distance from potential governance measures, otherwise available inside the hospital systems. Medical teams have to include in their decisions patients' preferences, the agreed upon free choice paradigm can contrast with hospital proposals, the digital divide can introduce troubles in terms of distant treatments, there can be such a problem of infrastructure constraints, like the accessibility, or difficulties in sensitization and informed consent. Other frictions could occur when the service is experienced effectively, as in the case when the care at home is delayed as consequence of previous errors in diagnosis, or when rapid readmissions are not possible/practicable. Nevertheless, this paper has presented a HaH implementation use case, testimonial to the fact that, notwithstanding issues of ethics, politics and policy ramifications, $\mathrm{HaH}$ is a viable system component in H-SES. As in the case of any other complex system implementation, there has to be a clear definition for fit for use and fit for purpose, which other works stipulate could bolster the ethical posture (Badr et al, 2021).

\section{CONCLUSION}

As a complex system, the H-SES must be dynamic, allowing for constant change but minimizing disruption in the outcome of the services. The robustness of the system must balance its flexibility to adapt, reconfigure in the face of changes in the environment, conditions and constraints.

In general, systems thinking studies have considered variety, variability and uncertainty as pillars for defining actions and interconnections among actors. Our use case reinforces this thinking by introducing examples of adaptation, and re-configurability. Additionally, principles of sustainablility through increased efficiency of healthcare service, effectiveness in resource usage, and patients' satisfaction were a central finding in our use case. This is a use case of exploiting technology to re-configure the Healthcare Service Ecosystem with structured coordination activities, patient and caregiver involvement, training of practitioners. While we can detect the contribution of the usual suspects in a technology implementation, this study underscores the importance of aligning to the quadruple aim of care, health, cost and meaning in work (Sikka et al, 2015).

Grounded in the works of contemporary scholars, this paper offers valuable insights for future implementation of similar $\mathrm{HaH}$ solutions that align with the quadruple aim principle. The modular design of the HaH technical solution was an important element in its success, demonstrating a real capacity to solve patient's problems and chronical difficulties. The system could be used by patients with different health conditions, in different setting, with or without the assistance of caregivers, with exchangeable modes to make an efficient and high quality healthcare service. 
Our use case was timely and effective in upholding the principles of the Quadruple Aim. The unwavering focus on patient experience, improving their outcome, lowering their risk and creating a service ecosystem where all actors are satisfied, costs are controlled and services are rendered effectively. The project was deemed a success as it has bolstered the value of $\mathrm{HaH}$ as a viable model of care. As a result, the ADD project was sanctioned and the local level, into a set of $\mathrm{HaH}$ services.

Nowadays, clinicians are leading service reconfiguration to cope with COVID-19, through learning new skills, adapting and exploiting new means of consultations. In other cases, hospitals have had to reconfigure their health services to reduce the workload of caregivers during the COVID-19 outbreak. Our use case can be a lesson for the adaptation of technology for patient empowerment allowing patients to interact with their care ecosystem while at their home.

\section{REFERENCES}

Al-Balushi, S., Sohal, A.S., Singh, J., Al Hajri, A., Al Farsi, Y.M., \& Al Abri, R. (2014). Readiness factors for lean implementation in healthcare settings-A literature review. J. Health Organ. Manag, 28, 135-153.

Al-Turjman, F., Nawaz, M.H., \& Ulusar, U.D. (2020). Intelligence in the Internet of Medical Things era: A systematic review of current and future trends. Computer Communications, 150, 644-660.

Arras, J.D., \& Dubler, N.N. (1994). Bringing the hospital home ethical and social implications of hightech home care. The Hastings Center Report, 24(5), S19-S28.

Badr, N., Drăgoicea, M., Walletzký, L., Carrubbo, L., \& Toli, A.M. (2021, January). Modelling for Ethical Concerns for Traceability in Time of Pandemic "Do no Harm" or "Better Safe than Sorry!". In Proceedings of the 54th Hawaii International Conference on System Sciences (p.1779).

Badr, N.G., Sorrentino, M., \& De Marco, M. (2018, September). Health Information Technology and Caregiver Interaction: Building Healthy Ecosystems. In International Conference on Exploring Service Science (pp. 316-329). Springer, Cham.

Badr, N.G., Sorrentino, M., De Marco, M., \& Fugini, M. (2019, June). Improving Interaction in Integrated Chronic Care Management. In 2019 IEEE 28th International Conference on Enabling Technologies: Infrastructure for Collaborative Enterprises (WETICE) (pp. 265-270). IEEE.

Baily, M.A., Bottrell, M., \& Lynn, J. (2007). Health care quality improvement: Ethical and regulatory issues. B. Jennings (Ed.). Hastings Center.

Barile, S., Polese, F., \& Carrubbo L. (2012a). Il cambiamento quale fattore strategico per la sopravvivenza delle organizzazioni imprenditoriali. In S. Barile, F. Polese, \& M. Saviano (Eds.), Immaginare l'Innovazione (pp. 1-35).

Barile, S., Polese, F., Saviano, M., \& Carrubbo L. (2016). Service Innovation in Translational Medicine. In T. Russo Spena, C. Mele, \& M. Nuutinen (Eds.), Innovation in Practices, Perspectives and Experiences (pp. 417-438). Springer.

Barile, S., Polese, F., Saviano, M., Carrubbo, L., \& Clarizia, F. (2012b). Service Research Contribution to Healthcare Networks' Understanding. In J. Mickelsson \& A. Helkkulla (Eds.), Innovative Service Perspectives. Hanken School of Economics, Hanken.

Bodenheimer, T., Lorig, K., Holman, H., \& Grumbach, K. (2002). Patient self-management of chronic disease in primary care. JAMA, 288, 2469-2475.

Bonadio, B., Huo, Z., Levchenko, A.A., \& Pandalai-Nayar, N. (2020). Global supply chains in the pandemic (No. w27224). National Bureau of Economic Research.

Breslow, L. (1992). Empowerment, not outreach: Serving the health promotion needs of the inner city. American Journal of Health Promotion, 7(1), 7.

Britt, T.W., Castro, C.A., \& Adler, A.B. (2005). Self-engagement, stressors, and health: A longitudinal study. Pers Soc Psychol Bull, 31(11), 1475-1486.

Budd, J., Miller, B.S., Manning, E.M., Lampos, V., Zhuang, M., Edelstein, M., . . Short, M.J. (2020). Digital technologies in the public-health response to COVID-19. Nature Medicine, pp. 1-10. 
Capunzo, M., Polese, F., Boccia, G., Carrubbo, L., Clarizia, F., \& De Caro, F. (2013). Advances in service research for the understanding and the management of service in healthcare networks. In E. Gummesson, C. Mele, \& F. Polese (Eds.), System Theory and Service Science: Integrating three perspectives in a new service agenda (pp. 14-17). Giannini, Napoli.

Caputo, F. (2018). Approccio Sistemico e Co-Creazione di Valore in Sanità. Edizioni Nuova Cultura: Roma, Italy.

Carrubbo, L., Bruni, R., Cavacece, Y., \& Moretta Tartaglione, A. (2015, September 12). Service System Platforms to improve value co-creation: Insights for Translational Medicine. In E. Gummesson, C. Mele, \& F. Polese (Eds.), System Theory and Service Science: Integrating three perspectives in a new service agenda. Napoli.

Carrubbo, L., Clarizia, F., Hysa, X., \& Bilotta, A. (2013). New "smarter" solutions for the healthcare complex service system. In E. Gummesson, C. Mele, \& F. Polese (Eds.), System Theory and Service Science: Integrating Three Perspectives in a New Service Agenda (pp. 14-17). Giannini, Napoli.

CDC. (2020). Using Telehealth to Expand Access to Essential Health Services during the COVID-19 Pandemic. Retrieved from https://cdc.gov/coronavirus/2019-ncov/hcp/telehealth.html

Ciasullo, M.V., Cosimato, S., \& Pellicano, M. (2017). Service innovations in the healthcare service ecosystem: A case study. Systems, 5(2), 37.

Cohen, S.R., Hassan, S.A., Lapointe, B.J., \& Mount, B.M. (1996). Quality of life in HIV disease as measured by the McGill quality of life questionnaire. Aids, 12, 1421-1427.

Crawford, M. (2017). Top 6 Robotic Applications in Medicine. ASME.ORG. Retrieved from https://aabme.asme.org/posts/top-6-robotic-applications-in-medicine

Finsterwalder, J., \& Kuppelwieser, V.G. (2020). Equilibrating resources and challenges during crises: A framework for service ecosystem well-being. Journal of Service Management.

Frow, P., McColl-Kennedy, J.R., Hilton, T., Davidson, A., Payne, A., \& Brozovic, D. (2014). Value propositions: A service ecosystems perspective. Marketing Theory, 14(3), 327-351.

Frow, P., McColl-Kennedy, J.R., Payne, A., \& Govind, R. (2019). Service ecosystem well-being: Conceptualization and implications for theory and practice. European Journal of Marketing

Grimm, C.A. (2020, March 23-27). Hospital Experiences Responding to the COVID-19 Pandemic: Results of a National Pulse Survey. U.S. HHS. OIG.

Gruman, J., Rovner, M.H., French, M.E., Jeffress, D., Sofaer, S., Shaller, D., \& Prager, D.J. (2010). From patient education to patient engagement: Implications for the field of patient education. Patient Education and Counseling, 78(3), 350-356.

Häring, I., Sansavini, G., Bellini, E., Martyn, N., Kovalenko, T., Kitsak, M., . . Linkov, I. (2017). Towards a generic resilience management, quantification and development process: General definitions, requirements, methods, techniques and measures, and case studies. In Resilience and Risk (pp. 21-80). Springer, Dordrecht.

Hwang, J., \& Christensen, C.M. (2008). Disruptive innovation in health care delivery: A framework for business-model innovation. Health Aff., 27, 1329-1335.

Ignone, G., Mossa, G., Mummolo, G., Pilolli, R., \& Ranieri, L. (2013). Increasing public healthcare network performance by de-hospitalization: A patient pathway perspective. Strateg. Outsourc. Int. J., 6, 85-107.

Leff, B. (2001). Acute? Care at home. The health and cost effects of substituting home care for inpatient acute care: A review of the evidence. J. Am. Geriatr. Soc., 49, 1123-1125.

Levine, D.M., Ouchi, K., Blanchfield, B., Saenz, A., Burke, K., Paz, M., \& Schnipper, J.L. (2020). Hospital-level care at home for acutely ill adults: A randomized controlled trial. Ann. Intern. Med., 172(2), 77-85.

Moazzami, B., Razavi-Khorasani, N., Moghadam, A.D., Farokhi, E., \& Rezaei, N. (2020). COVID-19 and telemedicine: Immediate action required for maintaining healthcare providers well-being. Journal of Clinical Virology, 104345. 
Moliner, M.A. (2009). Loyalty, perceived value and relationship quality in healthcare services. Journal of Service Management, 20(1), 76-97.

Nahum-Shani, I., Smith, S.N., Spring, B.J., Collins, L.M., Witkiewitz, K., Tewari, A., \& Murphy, S.A. (2018). Just-in-time adaptive interventions (JITAIs) in mobile health: key components and design principles for ongoing health behavior support. Annals of Behavioral Medicine, 52(6), 446-462.

P2PH. (n.d.). Pathways to Population Health (P2PH). IHI.ORG. Retrieved from http://www.ihi.org/

Plochg, T., Delnoij, D.M., Hoogedoorn, N.P., \& Klazinga, N.S. (2006). Collaborating while competing? The sustainability of community-based integrated care initiatives through a health partnership. BMC Health Services Research, 6(1), 1-14.

Polese, F., \& Carrubbo, L. (2016). Eco-Sistemi di Servizio in Sanità. G. Giappichelli (Ed.). Torino, Italy.

Polese, F., Carrubbo, L., Caputo, F., \& Sarno, D. (2018). Managing Healthcare Service Ecosystems: Abstracting a Sustainability-Based View from Hospitalization at Home (HaH) Practices. Sustainability, pp.1-15.

Restifo, N., Cirilli, F., Gastaldi, F., Fugini, M., Garavaglia, G., \& Chiantore, L. (2015). Health and social care services for frail people: ICT as a sustainable enabler of integrated care. In International conference eHealth (pp. 193-197).

Rodríguez Verjan, C., Augusto, V., Xie, X., \& Buthion, V. (2013). Economic comparison between Hospital at Home and traditional hospitalization using a simulation-based approach. J. Enterp. Inf. Manag., 26, 135-153.

Sikka, R., Morath, J.M., \& Leape, L. (2015). The quadruple aim: Care, health, cost and meaning in work.

Sofaer, S., \& Firminger, K. (2005). Patient perceptions of the quality of health services. In Annual Review of Public Health, 26, 513-559.

Sorrentino, M., Badr, N.G., \& De Marco, M. (2017, May). Healthcare and the co-creation of value: Qualifying the service roles of informal caregivers. In International conference on exploring services science (pp. 76-86). Springer, Cham.

Sorrentino, M., De Marco, M., \& Rossignoli, C. (2016, May). Health care co-production: Co-creation of value in flexible boundary spheres. In International conference on exploring services science (pp. 649-659). Springer, Cham.

Sweeney, J.C., Danaher, T.S., \& Mccoll-Kennedy, J.R. (2015). Customer effort in value cocreation activities improving quality of life and behavioral intentions of health care customers. Journal of Service Research, 18(3), 318-335.

Thornton, J. (2020). Clinicians are leading service reconfiguration to cope with covid-19. BMJ, p.369.

Wilson, M.G. (2018). Rapid Synthesis: Identifying the Effects of Home Care on Improving Health Outcomes, Client Satisfaction and Health System Sustainability (p.9). McMaster Health Forum: Hamilton, ON, Canada. 